\title{
Can CT Perfusion Guide Patient Selection for Treatment of Delayed Cerebral Ischemia?
}

\author{
Pina C. Sanelli ${ }^{1,2 *}$, Rachel Gold ${ }^{1}$, Nikesh Anumula ${ }^{1}$, Austin Ferrone ${ }^{1}$, Carl E. Johnson ${ }^{1}$, \\ Joseph P. Comunale ${ }^{1}$, Apostolos J. Tsiouris ${ }^{1}$, Howard Riina ${ }^{3}$, Halinder Mangat ${ }^{4}$, \\ Axel Rosengart ${ }^{4}$, Alan Z. Segal ${ }^{4}$ \\ ${ }^{1}$ Department of Radiology, Weill Cornell Medical College, New York, USA \\ ${ }^{2}$ Department of Public Health, Weill Cornell Medical College, New York, USA \\ ${ }^{3}$ Department of Neurological Surgery, Weill Cornell Medical College, New York, USA \\ ${ }^{4}$ Department of Neurology, Weill Cornell Medical College, New York, USA \\ Email: *pcs9001@med.cornell.edu
}

Received November 8, 2012; revised December 20, 2012; accepted January 4, 2013

\begin{abstract}
Purpose: To evaluate qualitative and quantitative CT perfusion (CTP) for different treatment options of delayed cerebral ischemia (DCI) in aneurysmal SAH. Methods: Retrospective study of consecutive SAH patients enrolled in a prospective IRB-approved clinical trial. Qualitative analysis of CTP deficits were determined by two blinded neuroradiologists. Quantitative CTP was performed using standardized protocol with region-of-interest placement sampling the cortex. DCI was assessed by clinical and imaging criteria. Patients were classified into treatment groups: 1) hypertension-hemodilution-hypervolemia (HHH); 2) intra-arterial (IA) vasodilators and/or angioplasty; 3) no treatment. Mean quantitative CTP values were compared using ANOVA pairwise comparisons. Receiver operating characteristic (ROC) curves, standard error (SE) and optimal threshold values were calculated. Results: Ninety-six patients were classified into three treatment groups; 21\% (19/96) HHH, 34\% (33/96) IA-therapy and 46\% (44/96) no treatment. DCI was diagnosed in 42\% (40/96); of which 18\% (7/40) received HHH, 80\% (32/40) IA-therapy, and 2\% (1/40) no treatment. CTP deficits were seen in 50\% (48/96); occurring in 63\% (12/19) HHH, 94\% (31/33) IA-therapy, and 11\% (5/44) no treatment. Presence of CTP deficits had $83 \%$ sensitivity, $89 \%$ specificity, $90 \%$ positive predictive and $81 \%$ negative predictive values for treatment. Mean quantitative CTP values revealed significant differences in CBF ( $<0.0001)$ and MTT ( $p=0.0001)$ amongst the treatment groups. ROC analysis revealed CBF with the highest accuracy of 0.82 (SE 0.04) for comparing treatment groups. Threshold analysis calculated CBF of $30 \mathrm{~mL} / 100 \mathrm{gm} / \mathrm{min}(89 \%$ specificity, $71 \%$ sensitivity) for determining treatment. Conclusion: These initial findings of significant differences in CTP deficits for different treatment groups suggest that CTP may have a potential role in guiding patient selection for treatment of DCI.
\end{abstract}

Keywords: CT Perfusion; Aneurysmal Subarachnoid Hemorrhage; Delayed Cerebral Ischemia; Treatment

\section{Introduction}

Delayed cerebral ischemia (DCI) is a serious complication following aneurysmal SAH, and is the leading cause of morbidity and mortality in this patient population. Its pathophysiology is complex and poorly understood leading to delayed diagnosis. However, proximal vasospasm is thought to partly contribute to DCI causing reduced cerebral blood flow (CBF) to the distal vascular territory [1-3]. DCI manifests as clinical deterioration typically developing between 4 - 14 days following the initial aneurysm rupture with symptoms of focal neurological impairment (such as hemiparesis, hemiplegia, aphasia, etc.), or a decrease of at least 2 points on the Glasgow Coma Scale. Early diagnosis and prompt treatment of DCI may

${ }^{*}$ Corresponding author. prevent its devastating sequelae of permanent neurologic deficits, cerebral infarction and death.

There are several treatment options for DCI typically used in clinical practice. Medical management with hypervolemic, hemodilution and hypertensive $(\mathrm{HHH})$ therapy is commonly used as the initial treatment. $\mathrm{HHH}$ is a systemic therapy used to improve global cerebral perfusion by increasing both blood pressure and flow to the brain. For patients that do not adequately respond to HHH therapy, more selective invasive intra-arterial (IA) therapy is performed to immediately dilate an artery in spasm using vasodilatory medications and/or angioplasty in order to improve the blood flow to a specific region of the brain. However, in some patients continuous close observation in the neuro-intensive care unit (N-ICU) setting is sufficient depending on the patient's clinical con- 
dition and considering the risks associated with both $\mathrm{HHH}$ and IA-therapy. The main complications that may occur with HHH are cerebral edema, aneurysm re-bleeding, pulmonary edema, and potential cardiac injury related to the aggressive use of pressor type medications [4]. Several important risks associated with IA-therapy include vascular injury (such as dissection and vessel rupture), cerebral infarction and death related to the arterial catheterization techniques and balloon angioplasty. Therefore, more accurate assessment of patients who require treatment of DCI is critical to improve patient selection in order to provide maximal treatment benefit and to minimize exposing patients to unnecessary serious risks.

Several methods are used for the diagnosis of DCI related to vasospasm, including clinical examination, neurologic monitoring devices, transcranial Doppler ultrasound (TCD), CTA, and DSA. Many institutions have also employed CT perfusion (CTP) to evaluate the hemodynamic status of the brain at the capillary level. CTP is a dynamic imaging study using cine scanning to capture the first pass of contrast through the brain to assess cerebral perfusion. The acquired dataset is post-processed using a deconvolution algorithm to construct the perfusion maps of CBF, cerebral blood volume (CBV) and mean transit time (MTT). The principle of CTP is that these perfusion parameters can be derived from the mathematical algorithm that describes the relationship between the arterial input, capillary flow and venous drainage. The arterial and venous time attenuation curves are obtained from the acquired dataset, representing the time series data of contrast through the brain. Two assumptions are made with CTP including that blood and contrast have the same hemodynamic properties and there is a linear relationship between contrast concentration in the brain with the measured Hounsfield units. There are several advantages of CTP compared with other perfusion imaging techniques, including its noninvasiveness, short acquisition time, widespread availability, and limited patient contraindications lending to its usefulness in this critically ill patient population. In the literature, several studies have reported the ability of CTP to detect impairment of cerebral perfusion thought to occur in the development of DCI and vasospasm [1-3,5]. However, not all perfusion deficits correlate with symptoms of DCI or arterial narrowing on imaging studies, indicating that not all perfusion deficits require treatment. In particular, the issue of which perfusion deficits require treatment with $\mathrm{HHH}$ and/or IA-therapy has not been fully explored. Thereby, further understanding of these CTP deficits is needed in order to improve patient selection for treatment of DCI. Quantitative differences in CTP deficits may assist in identifying patients earlier who require treatment, particularly in patients with limited clinical assessments, uncertain clinical findings, and discrepant clinical and imaging data, that may lead to delayed diagnosis and treatment.

The purpose of this study is to evaluate qualitative and quantitative CTP for different treatment options of DCI in aneurysmal SAH. Our hypothesis is that there are statistically significant differences in the CTP parameters in patients who are selected for different treatment regimens.

\section{Materials and Methods}

\subsection{Study Design}

A retrospective study of 104 consecutive aneurysmal SAH patients enrolled in a prospective clinical trial from December 2004 to December 2008 was performed. This study was approved by the institutional review board (IRB) and a health insurance portability and accountability act (HIPAA) waiver was obtained. Inclusion criteria were adult patients (18 years and older) with documented aneurysmal SAH at admission based on the initial noncontrast head CT, cerebrospinal fluid analysis, CTA and/ or DSA. Exclusion criteria were CTP exams performed after treatment of DCI or after cerebral infarction occurred. All subjects in the study underwent surgical clipping and/or endovascular coiling for aneurysm repair and were monitored in the N-ICU, as per usual standard-ofcare. Chart review was performed for the clinical and demographic characteristics of the study population, including age, gender, aneurysm location and treatment, and Hunt Hess grades on presentation. Treatment management decisions were based on all clinical and imaging data available, with the exception of CTP. Patients were categorized into three treatment groups as 1) medical HHH only; 2) IA-therapy; and 3) no treatment (close observation). Patients classified in the IA-therapy group did not adequately respond to $\mathrm{HHH}$ as the initial treatment.

The diagnosis of DCI was determined for each treatment group to assess appropriate classification of patients. DCI was determined in this cohort by incorporating the most relevant clinical and imaging outcome measures, as recommended by the recent consensus expert opinion [6]. Furthermore, Reichman et al. developed and evaluated a reference standard incorporating these outcomes measures to assess aneurysmal SAH patients applicable to clinical practice [7]. This reference standard consists of angiographic criteria for vasospasm based on the arterial luminal narrowing on DSA compared with the normal parent vessel and comparison with DSA performed on initial presentation. In this study, DSA interpretations were performed by two observers, one of two intervenetional neuroradiologists at our institution who performed the exam (with either 10 or 25 years experience) and a neuroradiologist blinded to all patient information (22 
years' experience). For disagreements, a third neuroradiologist (10 years' experience) independently reviewed the exam in a blinded fashion. In patients who did not have DSA performed, the following clinical and/or imaging criteria were used: a) clinical criteria-a permanent neurologic deficit on clinical examination, distinct from the deficit at baseline produced by the SAH event or surgical intervention, which was not attributable to other causes; b) imaging criteria-cerebral infarction on follow-up CT and/or MR imaging that occurred after day 4. This criterion has been used to effectively exclude primary brain damage from SAH and/or surgical intervenetion [8].

Clinical outcome measures were also determined for each treatment group to further assess appropriate classification of patients. At discharge from the N-ICU, patients were evaluated for a permanent neurologic deficit on physical examination and cerebral infarction on follow-up CT and/or MR imaging. Discharge status was also documented as home, rehabilitation care facility (acute rehabilitation or long-term skilled nursing), and death.

\subsection{CTP Scanning Protocol, Post-Processing and Data Collection}

CTP was performed during the typical time-period for DCI, between days 6 - 8 in asymptomatic patients and on the same day clinical deterioration occurred in symptomatic patients. There is a standard scanning protocol for CTP at our institution using GE Lightspeed or Pro-16 scanners (General Electric Medical Systems, Milwaukee, WI) with cine $4 \mathrm{i}$ scanning mode and 45 second acquisition at 1 rotation per second using $80 \mathrm{kVp}$ and $190 \mathrm{~mA}$. A scanning volume of $2.0 \mathrm{~cm}$ was used consisting of 4 slices at $5.0 \mathrm{~mm}$ thickness with its inferior extent selected at the level of the basal ganglia, above the orbits, to minimize radiation exposure to the lenses. Approximately $45 \mathrm{~mL}$ of nonionic iodinated contrast was administered intravenously at $5 \mathrm{~mL} / \mathrm{s}$ using a power injector with a 5-second delay.

Post-processing of the acquired images into cerebral blood flow (CBF), cerebral blood volume (CBV) and mean transit time (MTT) maps were performed on a GE Advantage Workstation using CTP software version 3.0 (General Electric Medical Systems). This software employs a deconvolution method, which is considered most accurate for low contrast injection rates [9]. The postprocessing technique was standardized for all patients according to recommended guidelines [10] with the arterial input function as the A2 segment of the anterior cerebral artery (ACA) [11] and venous function as the superior sagittal sinus.

The perfusion maps were qualitatively evaluated by two neuroradiologists (10 and 7 years experience) blinded to clinical and imaging data to determine the presence of perfusion deficits, defined as areas of reduced CBF and/ or prolonged MTT. Focal perfusion abnormalities due to the primary hemorrhagic event and surgical intervention, as identified on the acquired images from the CTP dataset, were not included as perfusion deficits related to DCI. After reviewing the images independently, consensus judgment was determined.

Quantitative analysis was conducted using a standardized method with contiguous regions-of-interest (ROI) placement, measuring $157 \mathrm{~mm}^{2}$, sampling the cerebral cortex. Each CTP slice had up to 24 ROIs distributed in the following territories: approximately 6 ROIs in ACA, 12 ROIs in middle cerebral artery (MCA) and 6 ROIs in posterior cerebral artery (PCA). CTP studies were analyzed blinded to all clinical and imaging data to limit test review bias.

\subsection{Statistical Analysis}

Only CTP exams performed at the diagnostic stage, prior to treatment for DCI and prior to cerebral infarction, were included in the analysis in order to minimize confounding bias as both treatment and infarction affect the cerebral perfusion maps. The incidence of qualitative CTP deficits in each treatment group was calculated. CTP test characteristics (sensitivity, specificity, positive and negative predictive values) were determined using 2 $\times 2$ table analysis with $95 \%$ confidence intervals (CI). Quantitative CTP data was analyzed by calculating the mean CBF, CBV and MTT and its standard deviation (SD) for the three treatment groups. For patients with focal perfusion deficits, ROIs within the affected region were isolated and arithmetic means were calculated. In patients without focal deficits, all ROIs for all 4 slice locations were included in the arithmetic means. In order to minimize the contribution of vascular pixels from large vessels, CBF values $>100 \mathrm{~mL} / 100 \mathrm{gm} / \mathrm{min}$ were excluded from the statistical analysis and not used in calculating the mean CBF, CBV and MTT, as this method has been published in the evaluation of ischemia [12]. The ROIs in the perfusion abnormalities due to the primary hemorrhagic event and/or surgical intervention, were not included in the statistical analysis. Normality of the distribution of continuous variables was assessed by plotting histograms and the Shapiro Wilks test. Comparison between the treatment groups was performed using ANOVA pairwise comparisons. Statistical significance was accepted at $\mathrm{p}<0.05$. Receiver operator characteristic (ROC) curves were generated for each CTP parameter and the area-under-the-curve (AUC) calculated to quantify the overall accuracy. Maximum-likelihood ROC models and standard error (SE) were fitted assuming a binormal distribution for the underlying latent variable. The threshold values for CBF, MTT and CBV 
were assessed using the northwest corner method, representing the point value on the ROC curve with the greatest discrimination ability between treatment groups. The statistical analysis was performed by a biostatistician using Stata version 10 and $\mathrm{R}$ version 2.7.2 software.

\section{Results}

\subsection{Study Population Characteristics}

A total of 96 aneurysmal SAH patients were included in the statistical analysis from the 104 patients enrolled in the prospective clinical trial. Eight patients were excluded for the following reasons: CTP exams were not performed prior to treatment for DCI $(n=4)$, CTP acquired data was not retrievable from the archives for post-processing in this study $(\mathrm{n}=3)$, and post-processing could not be performed due to severe motion degradation $(\mathrm{n}=1)$. The median age (range) was 49 years old (28 80 years) with a $73 \%$ (70/96) female predominance. Ninety-two percent (88/96) of the aneurysms were located in the anterior circulation. The treatment for aneurysm repair in this study population was 54\% (52/96) surgical clipping and 46\% (44/96) endovascular coiling procedures. The clinical Hunt Hess grades on presentation were 48\% (46/96) high grades 3, 4 and 5 and 52\% (50/96) low grades 1 and 2.

The study population was defined into three treatment groups; 20\% (19/96) of patients received $\mathrm{HHH}$ only, 34\% (33/96) underwent IA-therapy and 46\% (44/96) received no treatment (close observation).

\subsection{Qualitative CTP Analysis}

The median day CTP was performed was day 7 after aneurysm rupture. Qualitative CTP deficits were seen in $50 \%(48 / 96)$ of the study population, occurring in $95 \%$ (38/40) of patients with DCI and 18\% (10/56) without DCI. These CTP deficits were seen in 94\% (31/33) of patients who underwent IA-therapy, 63\% (12/19) who received $\mathrm{HHH}$, and $11 \%$ (5/44) who did not receive treatment. The test characteristics $(95 \% \mathrm{CI})$ for qualitative CTP deficits were 83\% (70\% - 91\%) sensitivity, 89\% (76\% - 95\%) specificity, 90\% (78\% - 95\%) positive predictive value, and $81 \%$ (68\% - 90\%) negative predictive value for determining patients requiring treatment.

\subsection{Quantitative CTP Analysis}

The mean quantitative values and standard deviation for CBF, CBV and MTT for each treatment group are shown in Table 1. There are statistically significant differences in the CBF and MTT values amongst the three treatment groups. Specifically, pairwise comparisons showed a statistically significant difference between the no treatment and IA-therapy groups for the CBF ( $<<0.0001)$, CBV ( $p=0.0343)$ and MTT $(p<0.0001)$. Furthermore, a statistically significant difference was also seen between the $\mathrm{HHH}$ and IA-therapy groups only for CBF ( $\mathrm{p}=$ 0.0406).

ROC curve analysis comparing the treatment and no treatment groups revealed that $\mathrm{CBF}$ and MTT had the highest overall accuracy of 0.82 (SE 0.04) and 0.81 (SE 0.05), respectively (Figure 1(a)). A similar trend is seen in the analysis comparing the $\mathrm{HHH}$ and IA-therapy groups with the highest overall accuracy for CBF of 0.72 (SE 0.07) and MTT of 0.69 (SE 0.08) (Figure 1(b)).

Threshold analysis was performed using the northwest corner method to determine the CTP value with the greatest discrimination ability between the treatment groups (Table 2).

\subsection{Clinical Outcomes}

DCI was diagnosed in 42\% (40/96) of patients according to the clinical and imaging criteria used in this study. In the IA-therapy group, 97\% (32/33) of patients were classified as DCI compared to $37 \%(7 / 19)$ in the $\mathrm{HHH}$ and $2 \%(1 / 44)$ in the no treatment groups. Since DCI was determined retrospectively using defined criteria for the purpose of this study, all treatment decisions were based on the clinical and imaging data available at the time of decision-making, with the exception of CTP.

Patient outcomes were evaluated using clinical and imaging criteria at the time of discharge from the N-ICU (Table 3). The IA-therapy group had the highest occurrence of permanent neurologic deficits and cerebral infarction, followed by the $\mathrm{HHH}$ and no treatment groups. The discharge status was also evaluated as a surrogate outcome measure (Table 4). A similar trend was also observed in the IA-therapy group having the worst outcomes with the highest mortality rate during hospitalization and the highest occurrence of patients requiring

Table 1. Mean quantitative CTP values and standard deviation for each treatment group.

\begin{tabular}{|c|c|c|c|c|}
\hline Mean (SD) & No treatment & HHH & IA & p-value \\
\hline CBF (mL/100 gm/min) & $40.6(11.1)$ & $33.2(15.4)$ & $24.4(11.9)$ & $<0.0001$ \\
\hline CBV (mL/100 gm) & $2.01(0.51)$ & $1.99(0.68)$ & $1.71(0.66)$ & 0.0825 \\
\hline MTT (sec) & $4.7(1.7)$ & $6.6(4.3)$ & $7.4(2.7)$ & 0.0001 \\
\hline
\end{tabular}



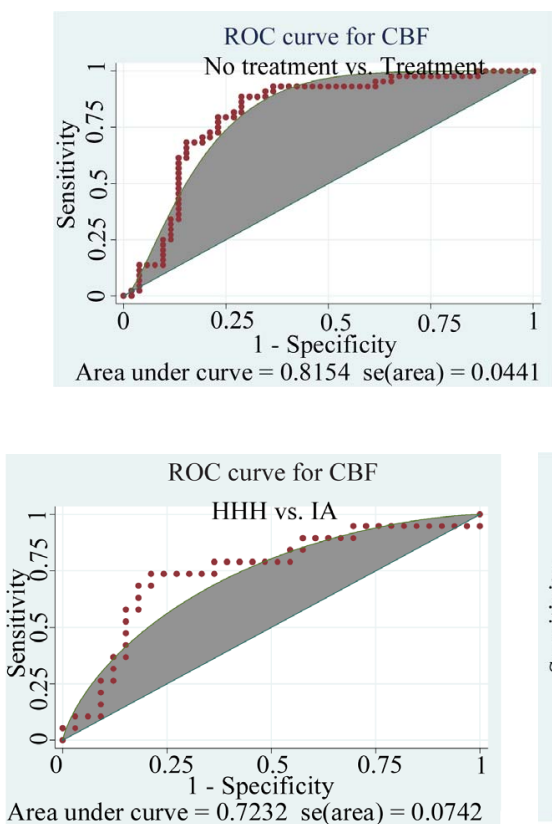

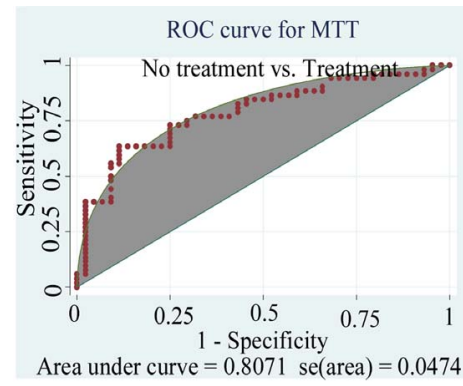

(a)

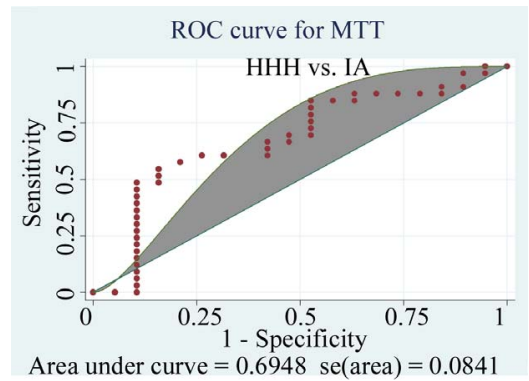

(b)
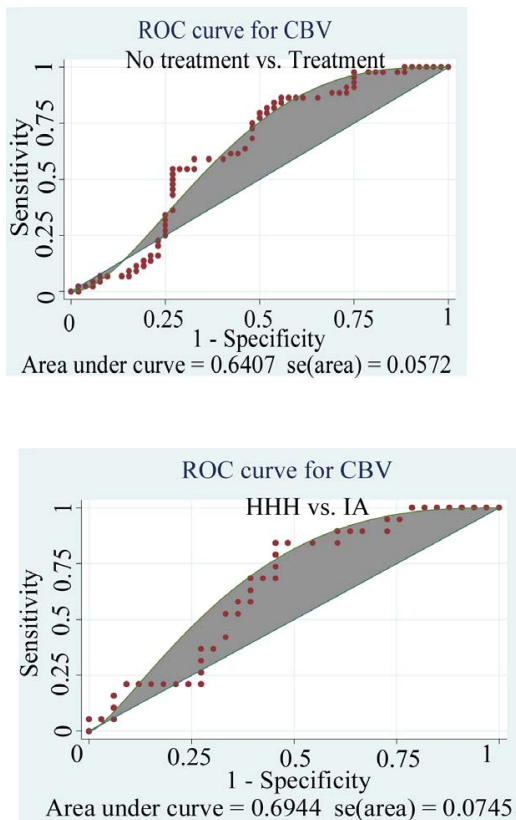

Figure 1. Receiver operator characteristic (ROC) curves of CBF, CBV and MTT for comparison of treatment groups.

Table 2. Threshold analysis for treatment options; (a) Treatment vs. no treatment; (b) HHH vs. IA-therapy.

(a)

\begin{tabular}{ccc}
\hline & Threshold & Specificity/Sensitivity \\
\hline CBF & 30 & $89 \% / 71 \%$ \\
MTT & 5.1 & $75 \% / 71 \%$ \\
CBV & 1.7 & $75 \% / 52 \%$ \\
\hline
\end{tabular}

(b)

\begin{tabular}{ccc}
\hline & Threshold & Specificity/Sensitivity \\
\hline CBF & 26 & $74 \% / 79 \%$ \\
MTT & 6.4 & $74 \% / 61 \%$ \\
CBV & 1.5 & $84 \% / 52 \%$ \\
\hline
\end{tabular}

Table 3. Clinical outcomes determined by clinical and imaging criteria.

\begin{tabular}{ccccc}
\hline & $\begin{array}{c}\text { Total } \\
(\mathbf{n}=\mathbf{9 6})\end{array}$ & $\begin{array}{c}\text { No } \\
\text { treatment } \\
(\mathbf{n}=\mathbf{4 4})\end{array}$ & $\begin{array}{c}\text { HHH } \\
(\mathbf{n}=\mathbf{1 9})\end{array}$ & $\begin{array}{c}\text { IA } \\
(\mathbf{n}=\mathbf{3 3})\end{array}$ \\
\hline $\begin{array}{c}\text { Functional } \\
\text { deficit/disability }\end{array}$ & $28 \%(27 / 96)$ & $11 \%(5 / 44)$ & $26 \%(5 / 19)$ & $52 \%(17 / 33)$ \\
$\begin{array}{c}\text { Cerebral } \\
\text { infarction }\end{array}$ & $14 \%(13 / 96)$ & $0 \%$ & $11 \%(2 / 19)$ & $33 \%(11 / 33)$ \\
\hline
\end{tabular}

rehabilitative care following discharge.

\section{Discussion}

DCI related to vasospasm is a complex entity involving
Table 4. Discharge status for treatment groups.

\begin{tabular}{ccccc}
\hline $\begin{array}{c}\text { Discharge } \\
\text { status }\end{array}$ & $\begin{array}{c}\text { Total } \\
(\mathbf{n}=\mathbf{9 6})\end{array}$ & $\begin{array}{c}\text { No } \\
\text { treatment } \\
(\mathbf{n}=\mathbf{4 4})\end{array}$ & $\begin{array}{c}\text { HHH } \\
(\mathbf{n}=\mathbf{1 9})\end{array}$ & IA (n= 33) \\
\hline $\begin{array}{c}\text { Home } \\
\text { Rehabilitative } \\
\text { care }\end{array}$ & $52 \%(50 / 96)$ & $68 \%(30 / 44)$ & $53 \%(10 / 19)$ & $30 \%(10 / 33)$ \\
$\begin{array}{c}\text { Acute } \\
\text { rehabilitation } \\
\text { Long-term } \\
\text { skilled }\end{array}$ & $10 \%(41 / 96)$ & $32 \%(14 / 44)$ & $42 \%(8 / 19)$ & $58 \%(19 / 33)$ \\
Death & $5 \%(5 / 96)$ & $9 \%(4 / 44)$ & $16 \%(3 / 19)$ & $6 \%(2 / 33)$ \\
\hline
\end{tabular}

delayed narrowing of the intracranial arteries that may lead to clinical deterioration, cerebral infarction and death. Current treatment options include medical management with $\mathrm{HHH}$ as the initial treatment to increase systemic blood pressure and flow in order to globally increase cerebral perfusion. Typically, if patients do not adequately respond to $\mathrm{HHH}$ then more invasive techniques, such as intra-arterial therapy with vasodilatory medications and/or angioplasty, are used to improve regional blood flow by selectively dilating individual cerebral arteries. Finally, close observation in the N-ICU may also be sufficient in some patients given the life-threatening complications associated with both $\mathrm{HHH}$ and IAtherapy. Therefore, more accurate patient selection for treatment of DCI is critical in order to provide maximal treatment benefit while minimizing exposure of patients to serious risks. 
In current practice, patient selection for treatment of DCI is based on clinical signs and symptoms coupled with imaging information of arterial narrowing for vasospasm. In recent years, CTP has also been used as a complimentary diagnostic tool for evaluation of DCI related to vasospasm. Specifically, qualitative CTP deficits have a high sensitivity and specificity [1-3,5,13-15] for vasospasm reported in $97 \%$ of patients with vasospasm compared to $24 \%$ without vasospasm [5]. Furthermore, Aralasmak et al. correlated CTP deficits and the degree of vessel narrowing with CTP deficits occurring in $83 \%$ of patients with severe vasospasm compared to $26 \%$ with mild-moderate and $15 \%$ without vasospasm [2]. Dankbaar et al. also demonstrated that the flow territory of the vessel with the most severe vasospasm corresponded with the least perfused region on CTP [16]. More recently, investigations have focused on using quantitative CTP and determining a threshold value. Several studies have reported CBF and MTT with the highest diagnostic accuracy for DCI $[3,5,16]$. A CBF threshold of $35 \mathrm{~mL} / 100 \mathrm{gm} / \mathrm{min}$ (90\% sensitivity, 68\% specificity) and MTT threshold of 5.5 sec (73\% sensitivity, 79\% specificity) have been reported [5]. Similarly, Dankbaar et al. described an MTT threshold of 5.9 sec for DCI [16]. Wintermark et al. also described the utility of CTP in the diagnosis and management of vasospasm suggesting that a CBF threshold of $39.3 \mathrm{~mL} / 100 \mathrm{gm} / \mathrm{min}$ represented the most accurate (94.8\%) indicator for endovascular therapy [3]. These results support the possibility that CTP-based criteria may be able to assist in differentiating patients according to the severity of DCI in attempts to guide patient selection for treatment.

A review of the literature is limited due to few studies performed in evaluating imaging based criteria for patient selection for treatment of DCI. In recent years, it is becoming more apparent that image-based criteria may have a role in patient selection for treatment of acute stroke. Turk et al. reported similar rates of good functional outcome and low rates of intracranial hemorrhage when endovascular treatment was performed based on CTP rather than time-guided selection $[17,18]$. This potential for image-based criteria may also have a role in aneurysmal SAH, particularly in patients with limited clinical examination, uncertain clinical findings, or discrepant clinical and imaging data. In the prospective management of patients, which perfusion deficits require treatment with $\mathrm{HHH}$ and/or IA-therapy still remains uncertain because not all perfusion deficits correspond to symptoms of DCI or arterial narrowing on imaging studies indicating that not all perfusion deficits require treatment (Figure 2). Thereby, further quantitative analysis of CTP deficits is needed to differentiate treatment groups in order to guide patient selection.

Our study focused on determining if there are qualitative

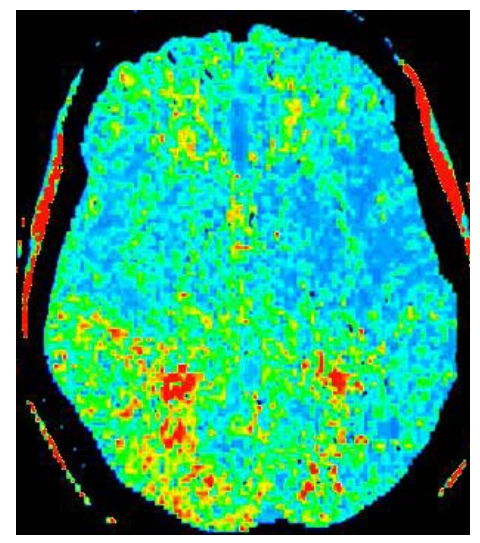

(a)

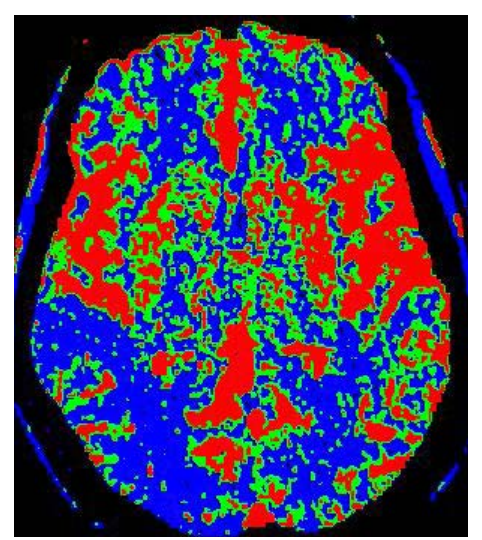

(b)

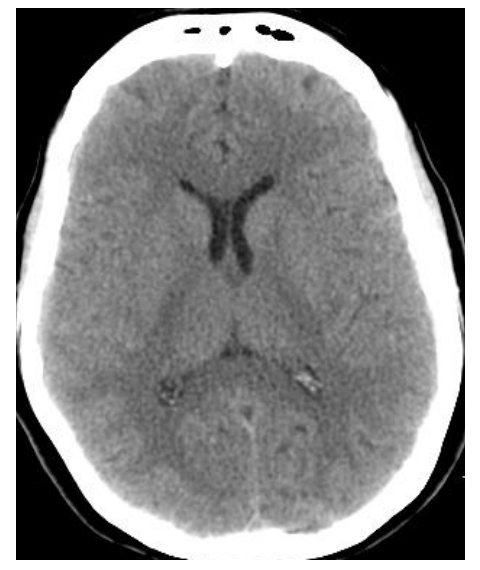

(c)

Figure 2. 57-year-old status post coil embolization of a right posterior communicating artery aneurysm. CT perfusion was performed on day 7 for the purposes of the study protocol and shows a focal perfusion deficit with elevated MTT (a) and reduced CBF (b) in the right MCAterritory in the parietal region. Quantitative evaluation of the perfusion deficit reveals a $\mathrm{CBF}$ of $37 \mathrm{~mL} / 100 \mathrm{gm} / \mathrm{min}$. The patient was asymptomatic and noncontrast CT (c) reveals no corresponding abnormality in this region. The patient did not receive treatment and was discharged home with resumption of normal daily activities. There was no evidence of a permanent neurologic deficit on clinical exam or cerebral infarction on follow-up CT imaging. 
and/or quantitative differences in CTP deficits for different treatment groups of DCI. The presence of a CTP deficit has a $90 \%$ positive predictive value for treatment. CTP deficits occurred in $94 \%$ (31/33) of the IA-therapy and $63 \%(12 / 19)$ of $\mathrm{HHH}$ groups compared to $11 \%(5 / 44)$ of the no treatment group. Further quantitative analysis revealed that CBF was the only perfusion parameter that showed a statistically significant difference in the pairwise comparisons of all three treatment groups. In addition, ROC curve analyses supported that CBF has the highest accuracy for determining the treatment group (Figure 1). Using this data, threshold analysis can be performed to help guide patient selection by determining the CBF value on the ROC curve with the greatest discrimination ability between treatment groups. In this study, a CBF threshold of $30 \mathrm{~mL} / 100 \mathrm{gm} / \mathrm{min}$ can be used as a guideline for a patient that should be carefully considered for treatment of DCI. Furthermore, a CBF threshold of $26 \mathrm{~mL} / 100 \mathrm{gm} / \mathrm{min}$ can be used as a rough guide for possibly considering patients who may also require more invasive techniques with IA-therapy. Given the proximity of these two threshold values, caution is emphasized when using absolute quantitative CTP values for the diagnosis and management of patients in clinical practice. The use of quantitative threshold values is limited in clinical practice due to differences in scanner equipment, techniques and post-processing methods employed. Importantly, standardization and validation of CTP methodology and post-processing techniques are necessary for its widespread implementation in SAH patients.

In this study, CTP deficits in the IA-therapy group had the most severely reduced CBF and prolonged MTT (Figure 3). The IA-therapy group had $97 \%$ of patients diagnosed with DCI, whereas the no treatment group had only $2 \%$ of patients with DCI. As expected, the IA- therapy group also had the worst outcomes with the highest mortality rate (12\%) and greatest percentage of patients with functional disability (52\%) and cerebral infarction (33\%). Whereas patients who had no treatment with close observation, the mean CBF and MTT values were within normal ranges. Improved outcomes were seen in the no treatment group with the highest rate of patients discharged home (68\%), lowest incidence of functional disability (11\%) and no cerebral infarction on follow-up CT/MR imaging. These findings support that there was appropriate classification of aneurysmal SAH patients in the different treatment groups.

We acknowledge several limitations in this study; including the retrospective study design limiting the outcomes data collection, specifically for clinical information. In addition, CTP provides limited brain coverage for evaluation of perfusion deficits. However, emerging CT scanner technology will continue to improve and

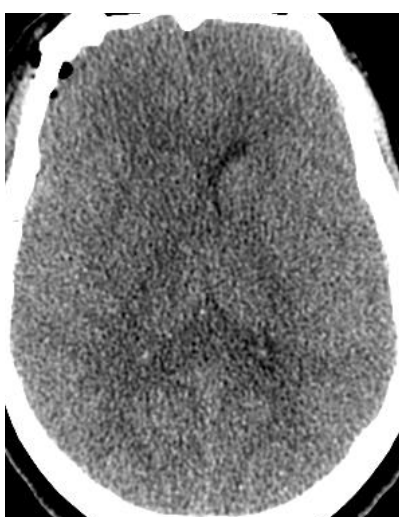

(a)

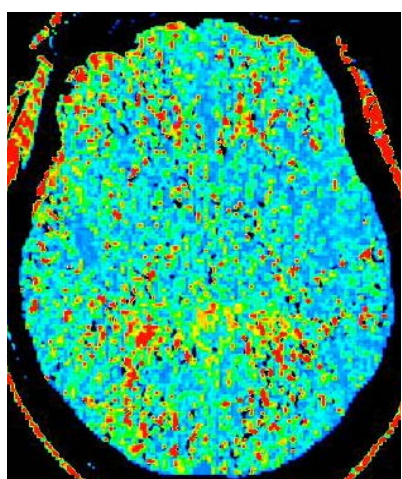

(b)

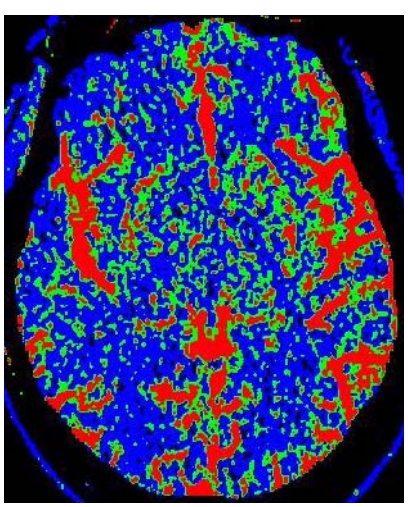

(c)

Figure 3. 35 year-old status post surgical clipping of a right posterior communicating artery aneurysm and developed new left-sided upper extremity hemiparesis and pronator drift on day 6. Noncontrast CT (a) demonstrates no new hemorrhage or hydrocephalus to explain the patient's symptoms. However, there is early hypodensity involving the right basal ganglia. CT perfusion performed the same day shows a large perfusion deficit in the right MCA territory in the frontal and parietal regions and focal perfusion deficit in the left ACA territory with elevated MTT (b) and reduced CBF (c). Quantitative evaluation reveals a CBF of $18 \mathrm{~mL} / 100 \mathrm{gm} / \mathrm{min}$ in the right MCA and CBF of $17 \mathrm{~mL} /$ $100 \mathrm{gm} / \mathrm{min}$ in the left ACA perfusion deficits. The patient received HHH therapy and also underwent IA-therapy with verapamil and angioplasty of the right MCA and left ACA. Patient developed cerebral infarction in the right anterior temporal lobe and basal ganglia. 
provide broader coverage for which this data may be applicable. Another limitation is that the region of CTP imaging was not coordinated with the location of the arterial narrowing on DSA or region referable to symptoms. Therefore, it is conceivable that this region may not have been imaged on the CTP or possibly averaged with a larger region of normal perfusion, resulting in increased false negatives and lower accuracy in our study. This method was not used in order to reduce work-up and observer bias by not having knowledge of the clinical exam and DSA results prior to CTP scanning and interpretation.

\section{Conclusion}

There is a clinical need to more accurately select aneurysmal SAH patients for treatment of DCI in order to provide maximal treatment benefit while minimizing patient exposure to serious complications. Critically ill and comatose patients with limited clinical examinations, uncertain clinical findings, and discrepant clinical and imaging data remain challenging to assess for treatment. CTP provides additional information regarding hemodynamic disturbance in the brain. Qualitative CTP deficits have a $90 \%$ positive predictive value for determining patients who require treatment. Since it remains uncertain which perfusion deficits require $\mathrm{HHH}$ and/or IAtherapy, further evaluation with quantitative analysis is needed to differentiate treatment groups. In this study, CBF showed statistically significant differences for the different treatment groups. CBF also had the highest accuracy and discrimination ability on the ROC curve for determining treatment groups using threshold analysis. These preliminary findings support continued work in this field with larger prospective clinical trials as CTP may have a role in guiding patient selection for treatment of DCI by its quantitative evaluation of perfusion deficits.

\section{Acknowledgements}

This publication was made possible by Grant Number 5K23NS058387-02 from the National Institute of Neurological Disorders and Stroke (NINDS), a component of the National Institutes of Health $(\mathrm{NIH})$. Its contents are solely the responsibility of the authors and do not necessarily represent the official view of NINDS or NIH.

\section{REFERENCES}

[1] J. W. Dankbaar, N. K. de Rooij, B. K. Velthius, C. J. Frijns, G. J. Rinkel and I. C. van der Schaaf, "Diagnosing Delayed Cerebral Ischemia with Different CT Modalities in Patients with Subarachnoid Hemorrhage with Clinical Deterioration,” Stroke, Vol. 40, No. 11, 2009, pp. 34933498. doi:10.1161/STROKEAHA.109.559013
[2] A. Aralasmak, M. Akyuz, C. Ozkaynak, T. Sindel and R. Tuncer, "CT Angiography and Perfusion Imaging in Patients with Subarachnoid Hemorrhage: Correlation of Vasospasm to Perfusion Abnormality,” Neuroradiology, Vol. 51, No. 2, 2009, pp. 85-93. doi:10.1007/s00234-008-0466-7

[3] M. Wintermark, N. U. Ko, W. S. Smith, S. Liu, R. T. Higashida and W. P. Dillon, "Vasospasm after Subarachnoid Hemorrhage: Utility of Perfusion CT and CT Angiography on Diagnosis and Management,” AJNR American Journal of Neuroradiology, Vol. 27, No. 1, 2006, pp. 26-34.

[4] J. Sen, A. Belli, H. Albon, L. Morgan, A. Petzold and N. Kitchen, "Triple-H Therapy in the Management of Aneurysmal Subarachnoid Haemorrhage,” Lancet Neurology, Vol. 2, No. 10, 2003, pp. 614-621. doi:10.1016/S1474-4422(03)00531-3

[5] P. C. Sanelli, I. Ugorec, C. E. Johnson, J. Tan, A. Z. Segal, M. Fink, et al., "Using Quantitative CT Perfusion for Evaluation of Delayed Cerebral Ischemia Following Aneurysmal Subarachnoid Hemorrhage,” AJNR American Journal of Neuroradiology, Vol. 32, No. 11, 2011, pp. 2047-2053. doi:10.3174/ajnr.A2693

[6] M. D. I. Vergouwen, M. Vermeulen, J. van Gijn, G. J. Rinkel, E. F. Wijdicks, J. P. Muizelaar, et al., "Definition of Delayed Cerebral Ischemia After Aneurysmal Subarachnoid Hemorrhage as an Outcome Event in Clinical Trials and Observational Studies: Proposal of a Multidisciplinary Research Group,” Stroke, Vol. 41, No. 10, 2010, pp. 2391-2395. doi:10.1161/STROKEAHA.110.589275

[7] M. B. Reichman, E. D. Greenberg, R. L. Gold and P. C. Sanelli, "Developing Patient-Centered Outcome Measures for Evaluating Vasospasm in Aneurysmal Subarachnoid Hemorrhage,” Academic Radiology, Vol. 16, No. 5, 2009, pp. 541-545. doi:10.1016/j.acra.2009.01.018

[8] J. A. Frontera, A. Fernandez, J. M. Schmidt, J. Claassen, K. E. Wartenberg, N. Badjatia, et al., "Defining Vasospasm after Subarachnoid Hemorrhage: What Is the Most Clinically Relevant Definition?” Stroke, Vol. 40, No. 6, 2009, pp. 1963-1968. doi:10.1161/STROKEAHA.108.544700

[9] M. Wintermark, P. Maeder, J. P. Thiran, P. Schnyder and R. Meuli, "Quantitative Assessment of Regional Blood Flows by Perfusion CT Studies at Low Injection Rates: A Critical Review of the Underlying Theoretical Models," European Radiology, Vol. 11, No. 7, 2001, pp. 12201230. doi:10.1007/s003300000707

[10] P. C. Sanelli, M. H. Lev, J. D. Eastwood, R. G. Gonzalez and T. Y. Lee, “The Effect of Varying User-Selected Input Parameters on Quantitative Values in CT Perfusion Maps,” Academic Radiology, Vol. 11, No. 10, 2004, pp. 1085-1092. doi:10.1016/j.acra.2004.07.002

[11] M. Wintermark, B. C. Lau, J. Chien and S. Arora, “The Anterior Cerebral Artery Is an Appropriate Arterial Input Function for Perfusion-CT Processing in Patients with Acute Stroke,” Neuroradiology, Vol. 50, No. 3, 2008, pp. 227-236. doi:10.1007/s00234-007-0336-8

[12] B. D. Murphy, A. J. Fox, D. H. Lee, D. J. Sahlas, S. E. Black, M. J. Hogan, et al., "Identification of Penumbra 
and Infarct in Acute Ischemic Stroke Using Computed Tomography Perfusion-Derived Blood Flow and Blood Volume Measurements,” Stroke, Vol. 37, No. 7, 2006, pp. 1771-1777. doi:10.1161/01.STR.0000227243.96808.53

[13] S. Binaghi, M. L. Colleoni, P. Maeder, A. Uske, L. Regli, A. R. Dehdashti, et al., "CT Angiography and Perfusion CT in Cerebral Vasospasm after Subarachnoid Hemorrhage,” AJNR American Journal Neuroradiology, Vol. 28, No. 4, 2007, pp. 750-758.

[14] M. Wintermark, W. P. Dillon, W. S. Smith, B. C. Lau, S. Chaudhary, S. Liu, et al., "Visual Grading System for Vasospasm Based on Perfusion CT Imaging: Comparisons with Conventional Angiography and Quantitative Perfusion CT," Cerebrovascular Disease, Vol. 26, No. 2, 2008, pp. 163-170. doi:10.1159/000139664

[15] J. W. Dankbaar, M. Rijsdijk, I. C. van der Schaaf, B. K. Velthuis, M. J. Wermer and G. J. Rinkel, "Relationship between Vasospasm, Cerebral Perfusion, and Delayed Cerebral Ischemia after Aneurysmal Subarachnoid Hemorrhage,” Neuroradiology, Vol. 51, No. 12, 2009, pp. 813-

\section{Abbreviations}

CTP: computed tomography perfusion

SAH: subarachnoid hemorrhage

DCI: delayed cerebral ischemia

CBF: cerebral blood flow

CBV: cerebral blood volume

MTT: mean transit time

$\mathrm{HHH}$ : hypertension hemodilution hypervolemia

IA: intra-arterial

DSA: digital subtraction angiography

TCD: transcranial doppler ultrasound

CTA: computed tomography angiography
819. doi:10.1007/s00234-009-0575-y

[16] J. W. Dankbaar, N. K. de Rooij, M. Rijsdijk, B. K. Velthuis, C. J. Frijns, G. J. Rinkel, et al., "Diagnostic Threshold Values of Cerebral Perfusion Measured with Computed Tomography for Delayed Cerebral Ischemia after Aneurysmal Subarachnoid Hemorrhage," Stroke, Vol. 41, No. 9, 2010, pp. 1927-1932. doi:10.1161/STROKEAHA.109.574392

[17] A. Turk, J. A. Magarik, I. Chaudry, R. D. Turner, J. Nicholas, C. A. Holmstedt, et al., "CT Perfusion-Guided Patient Selection for Endovascular Treatment of Acute Ischemic Stroke is Safe and Effective," Journal of Neurointerventional Surgery, Vol. 4, No. 4, 2012, pp. 261-265.

[18] A. S. Turk, J. A. Magarik, D. Frei, K. M. Fargen, I. Chaudry, C. A. Holmstedt, et al., "CT Perfusion-Guided Patient Selection for Endovascular Recanalization in Acute Ischemic Stroke: A Multicenter Study,” Journal of Neurointerventional Surgery, 2012, (Epub ahead of print) in PubMed.

MR: magnetic resonance

ACA: anterior cerebral artery

MCA: middle cerebral artery

PCA: posterior cerebral artery

N-ICU: neuro-intensive care unit

ROI: region of interest

ROC: receiver operator characteristic

AUC: area under curve

ANOVA: analysis of variance

SE: standard error

SD: standard deviation

$\mathrm{CI}$ : confidence interval 